\title{
A critical look at quantum diffusion and some of its interesting aspects
}

\author{
S. Mukhopadhyay ${ }^{1, a}$, M. Saglam ${ }^{2}$, and A. Chatterjee $3, \mathrm{~b}$ \\ 1 Department of Physics, Bilkent University, Bilkent, Ankara, Turkey \\ 2 Department of Physics, Faculty of Sciences, Ankara University, 06100 Tandogan-Ankara, Turkey \\ 3 Department of Physics, Bilkent University, 06800 Bilkent, Ankara, Turkey
}

Received 17 April 2007/ Received in final form 4 July 2007

Published online 5 October 2007 - (c) EDP Sciences, Società Italiana di Fisica, Springer-Verlag 2007

\begin{abstract}
A fresh and critical look has been given to the long-time behaviour of the quantum diffusion problem and a marginally more accurate solution has been obtained as compared to the one reported in the recent literature. Attempt has also been made to bring out a few interesting generic features of this problem which may have important bearing on real systems in the quantum domain.
\end{abstract}

PACS. 03.65.-w Quantum mechanics - 02.40.Xx Singularity theory - 71.23.Ft Quasicrystals $-71.30 .+\mathrm{h}$ Metal-insulator transitions and other electronic transitions

The long-time dynamical behaviour of wave packets of quantum systems is essentially a diffusion problem but in the quantum regime and therefore can be termed as quantum diffusion in a more general sense which includes on one hand the classical diffusion together with super-diffusive regime leading to ballistic motion and on the other the sub-diffusive regime leading to localization. There have been several investigations [1-11] on these issues primarily because of their importance in lowdimensional systems such as carbon nanotubes and several other systems and various interesting aspects of this problem have been studied in great detail in recent years. However, still, quite a lot of issues have remained unanswered and these require critical investigations to test the veracity of the fundamental quantum mechanical ideas at the laboratory level. Recently, Zhong et al. [12] have studied the shape of a quantum diffusive front and have shown that after an initial transient the wave packet front is described by a stretched exponential. They have provided an analytical derivation for the stretched exponential expression of the probability distribution using the memory function formalism of quantum dynamics, and have uncovered a universal relation between the shape exponent and the diffusion constant. They have demonstrated their results through numerical work on one-dimensional quasi-periodic systems and the three-dimensional Anderson model of disorder. They have also applied their re-

\footnotetext{
${ }^{\text {a }}$ On leave from Shadam Institute of P.G. Studies Khairatabad, Hyderabad, India.

b On leave from School of Physics, University of Hyderabad, Hyderabad, India.

e-mail: ashak@fen.bilkent.edu.tr
}

sults to some experimental results and have shown that their solution describes the experimental data pretty well. This work is in essence complementary to the work of Ketzmerich et al. [11] which is valid at smaller length scales. In the present paper we will give a fresh look at the quantum diffusion problem as studied in [12] and make an attempt to generalize their results and improve their analysis to obtain a more accurate solution and discuss the range of its validity. We shall also bring out a few more interesting features of this problem that have not been studied so far and that may have some important bearing on real systems.

The nontrivial time-evolution of any lattice model is given in the Schrödinger picture by the time-dependent Schrödinger equation

$$
\iota \hbar \frac{\partial \Psi(n, t)}{\partial t}=[T(n)+V(n)] \Psi(n, t)
$$

where $T(n, t)$ and $V(n, t)$ are respectively the kinetic and potential energies at the lattice site $n$ and at time $t$. Equation (1) can be rewritten as

$$
\iota \hbar \frac{\partial \Psi(n, t)}{\partial t}=V(n) \Psi(n, t)+\sum_{n^{\prime}} h\left(n, n^{\prime}\right) \Psi\left(n^{\prime}, t\right),
$$

where $h\left(n, n^{\prime}\right)=\left\langle n^{\prime}\left|T\left(n^{\prime}\right)\right| n\right\rangle$ is the nearest neighbour hopping integral. Given any lattice potential $V(n)$ one can integrate equation (2) numerically to obtain $\Psi(n, t)$ and finally calculate the probability $P(n, t)=|\Psi(n, t)|^{2}$ which gives the spreading of the wave packet pertaining to the system. Zhong et al. [12] have obtained $P$ as a function of $x$ for different model systems and have shown that the 
probability distribution $P(x, t)$ at long time is described by stretched exponential with a time-dependent exponent which has a universal relation with the scaling exponent of the spreading width. They have also given an analytical expression for $P(x, t)$ which fits pretty well with the numerical results obtained for the Fibonici chain and the three-dimensional Anderson model and with the experimental results for the quantum kicked rotor. We shall in the following go through their derivation rather critically and in the process make an attempt to modify their expression and point out the more accurate condition under which their expression should be valid.

The generalized master equation (GME) for the probability distribution is given in the memory function formalism by [12]

$$
\iota \hbar \frac{\partial P(n, t)}{\partial t}=\int d t^{\prime} \sum_{n^{\prime}} W\left(n, n^{\prime}, t-t^{\prime}\right) \Delta P\left(n^{\prime}, n, t^{\prime}\right),
$$

where $\Delta P\left(n^{\prime}, n, t^{\prime}\right)=P\left(n^{\prime}, t^{\prime}\right)-P\left(n, t^{\prime}\right)$ and $W\left(n, n^{\prime}, t-\right.$ $\left.t^{\prime}\right)$ is called the memory function. Another way to handle the problem would be to find out the Green function of the problem and then write,

$$
\Psi\left(n^{\prime}, t^{\prime}\right)=\sum_{n} G\left(n^{\prime}, t^{\prime}, n, t\right) \Psi(n, t) .
$$

It may be easier to work in the continuum limit and hence Zhong et al. have made a coarse grained averaging over the position $n$ and written the GME in terms of the coarse grained position $x$. After a gradient expansion of $\Delta P$ in $\Delta x$ and assuming a slow variation of density with space and homogeneity in time and isotropic diffusion, they have obtained

$$
\iota \hbar \frac{\partial P(x, t)}{\partial t}=\int d t^{\prime} I\left(t-t^{\prime}\right)\left(\frac{\partial^{2} P}{\partial x^{2}}\right)_{\Delta x=0},
$$

where

$$
I(t)=(1 / 2) \Sigma_{\Delta x} g(\Delta x, t)(\Delta x)^{2},
$$

with $g(\Delta x, t)$ being the coarse-grained average of the memory function. Defining Laplace transforms,

$$
\begin{aligned}
\tilde{P}(x, s) & =\int d t P(x, t) e^{-t s}, \\
\tilde{I}(x, s) & =\int d t I(t) e^{-t s},
\end{aligned}
$$

and the corresponding inverse transforms, one gets,

$$
\frac{\partial^{2} \tilde{P}(x, s)}{\partial x^{2}}=f^{2}(s) \tilde{P}(x, s),
$$

where $f(s)=\sqrt{ }[s / \tilde{I}(s)]$. The solution of equation (9) is simple and straight-forward integration gives,

$$
\tilde{P}(x, s)=B(s) e^{-|x| f(s)},
$$

where the normalization constant $B(s)=f(s) / 2$. The probability $P(x, t)$ is given by,

$$
P(x, t)=\frac{1}{4 \pi \iota} \int_{\delta-\iota \infty}^{\delta+\iota \infty} d s f(s) e^{[s t-|x| f(s)]} .
$$

Stipulating $f(s)=f_{0} s^{\beta}$ in the limit $s \rightarrow 0$ and using the stationary phase approximation we get,

$$
\beta|x| f_{0} s^{\beta}-s t-\beta=0,
$$

where $0<\beta<1$ and we are looking for the long-time behaviour for which $\beta / t \ll 1$. Also, we have the condition that $s \rightarrow 0$ and therefore solving the above equation becomes a tricky problem. However if we can assume that $\beta \ll s / t$ which could be plausible in the long time limit, then we get the same solution for $s$ as obtained by taking $\beta / t \ll 1$ i.e.,

$$
s \equiv s_{0}=\left[\frac{\beta|x| f_{0}}{t}\right]^{\gamma},
$$

where

$$
\gamma=\frac{1}{1-\beta}
$$

but the range of $x$ is now different. We now have,

$$
f_{0}|x| \gg\left[(t / \beta)^{\beta}\right]
$$

instead of Zhong et al's condition [12], $|x|>t^{\beta}$. Of course, as in the case of Zhong et al. we shall still have $|x|<t$. So the range of $\mathrm{x}$ for which our solution for $s$ will hold is:

$$
\frac{t^{\beta}}{f_{0} \beta^{\beta}} \ll|x|<t
$$

One could also solve the equation for $s$ by assuming $s t \ll \beta$ and this will give the small time behaviour of the system in which we have no interest at the moment. It is possible to systematically improve the solution of $s$ by an iterative procedure but we shall refrain from any such complicated solutions here. The probability is thus given by

$$
P(x, t)=A e^{-\left(\frac{|x|}{\omega}\right)^{\gamma}+\beta \ln \left(\frac{|x|}{t}\right)^{\gamma}},
$$

where

$$
w=\left[\frac{t^{\beta}}{f_{0} \beta^{\beta}(1-\beta)^{(1-\beta)}}\right] .
$$

It is clear that the present solution is different from that of Zhong et al. However, since $|x|<t$ but larger than $t^{\beta} /\left[f_{0} \beta^{\beta}\right]$, the second term in the exponential of $P(x, t)$ will be generally smaller than the first term and in that approximation one would get the same stretched exponential as obtained by Zhong et al. [12]. But the important point to note is that the log-term has a positive effect on the stretching of the exponential since $|x|<t$ and in the case when $\beta$ is close to 1 so that $\beta \times \gamma=\beta /(1-\beta)$ can become substantially large and then if $f_{0}$ is sufficiently small, the present solution can give a significant modification to the result of Zhong et al. For example, for a system with a very large shape exponent $\gamma(\beta \rightarrow 1)$ for the diffusion front i.e., in the ballistic limit and for small $f_{0}$, the present theory predicts a faster dynamics as compared to what suggested in [12]. Furthermore, the present solution suggests a slightly different range of $x$ from that of Zhong et al. In Table 1 we briefly summarize the results. We would like to emphasis that although the present solution 
Table 1. The probability $P(x, t)$ together with the range of the parameter values for its validity. The first two rows are our results while the third row gives the result of Zhong et al.[12].

\begin{tabular}{cccc}
\hline$t$ & $\beta=\frac{f(s)}{f_{0} \ln s}$ & $x$ & $P(x, t)$ \\
\hline$t \gg \beta$ & $0<\beta<1$ & $\frac{t^{\beta}}{f_{0} \beta^{\beta}} \ll x<t$ & $P \sim e^{-(|x| / \omega)^{\gamma}+\beta \ln (|x| / t)^{\gamma}}$ \\
$t \gg \beta$ & $0<\beta \ll 1$ & $\frac{t^{\beta}}{f_{0} \beta^{\beta}} \ll x<t$ & $P \sim e^{-(|x| / \omega)^{\gamma}}$ \\
$t \gg \beta$ & $0<\beta \leq 1$ & $t^{\beta}<x<t$ & $P \sim e^{-(|x| / \omega)^{\gamma}}$ \\
\hline
\end{tabular}

for the probability looks only marginally better than that of [12], it can lead to large deviations when moments and other physical quantities are considered.

In what follows, we would like to consider a few generic features instead of considering any specific examples. The normalization of the probability gives

$$
A=\frac{1}{2}\left(\frac{\gamma}{\omega}\right)\left(\frac{t}{\omega}\right)^{\beta \gamma}
$$

Straight-forward calculation of $\left\langle x^{2}\right\rangle$ immediately gives,

$$
\left\langle x^{2}\right\rangle=\Gamma(3-2 \beta)\left[\frac{t^{\beta}}{\left(f_{0} \beta^{\beta}(1-\beta)^{(1-\beta)}\right)}\right]^{2}
$$

in contrast to the result

$$
\left\langle x^{2}\right\rangle=\frac{\Gamma(3-3 \beta)}{\Gamma(1-\beta)}\left[\frac{t^{\beta}}{\left(f_{0} \beta^{\beta}(1-\beta)^{(1-\beta)}\right)}\right]^{2}
$$

obtained with the solution of [12]. One can immediately see that for $\beta=1 / 2$, both the equations (20) and (21) describe the classical diffusion, but the present solution yields a result that is twice the value given by Zhong et al. [12]. The classical diffusion constant is now given by $\left[2 / f_{0}^{2}\right]$. In Figures 1 and 2 we show the bahaviour of the moment $\left\langle x^{2}\right\rangle$ as a function of $t$ for a few values of $\beta$.

It is clear that the growth of the moment increases with increasing $\beta$, more so at large $t$. In Figures $3-5$ we compare our results for $\left\langle x^{2}\right\rangle$ with those of [12] for different values of $\beta$ corresponding to localization, diffusive, and ballistic regimes. It is clearly evident that our marginally improved solution for probability density leads to enormous deviations in the bahaviour of the moment $\left\langle x^{2}\right\rangle$ as compared to that obtained from the result of [12] for all ranges of $\beta$ at large $t$ which is the region of interest in the present paper. At small $t$ however the disagreement becomes smaller and smaller as $\beta$ increases.

It is natural to calculate the $\mathrm{k}$-th moment. It is clear that odd moments will vanish and the even moments can be written as:

$$
\begin{aligned}
\left\langle x^{k}\right\rangle & =2 A \int_{0}^{\infty} x^{k} e^{-\left[\left(\frac{x}{w}\right)^{\gamma}+\ln \left(\frac{x}{t}\right)^{\gamma \beta}\right]} d x \\
& =\frac{2 A}{t^{\gamma \beta}} \int_{0}^{\infty} x^{k+\gamma \beta} e^{-\left(\frac{x}{w}\right)^{\gamma}} d x .
\end{aligned}
$$

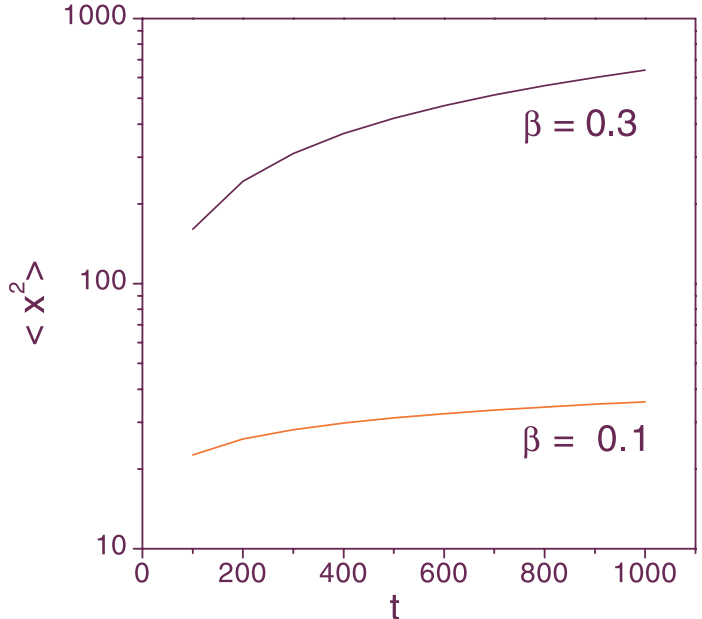

Fig. 1. The second moment as a function of time $t$ for $\beta=0.1$ and $\beta=0.3$.

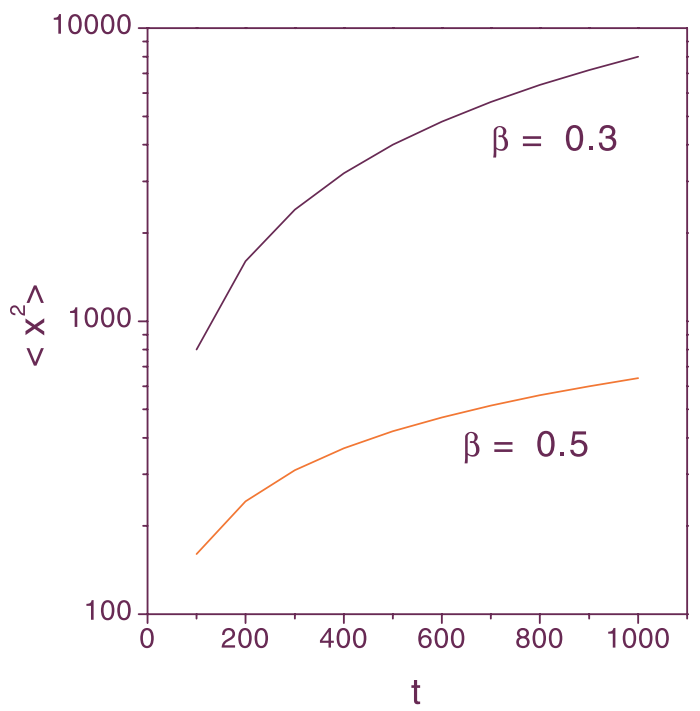

Fig. 2. The second moment as a function of time $t$ for $\beta=0.3$ and $\beta=0.5$.

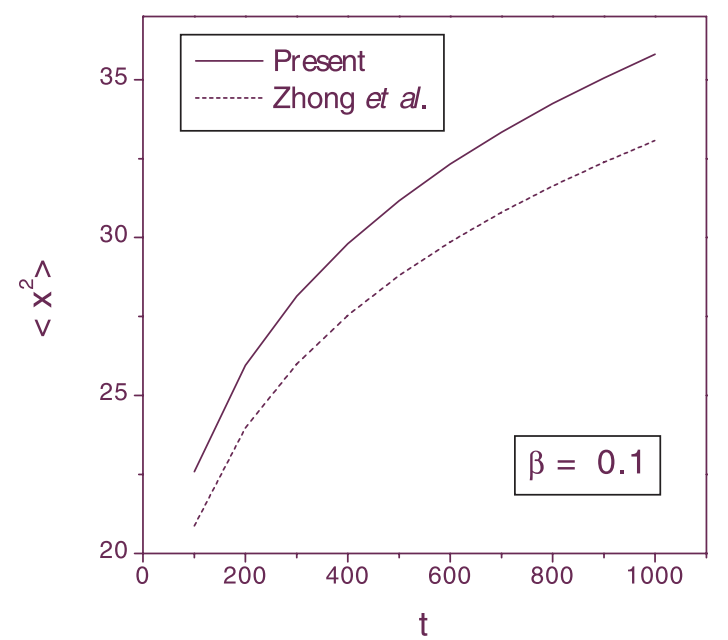

Fig. 3. The second moment as a function of time $t$ for $\beta=0.5$. The solid line gives our results while the dashed curve refers to the results of Zhong et al. [12]. 


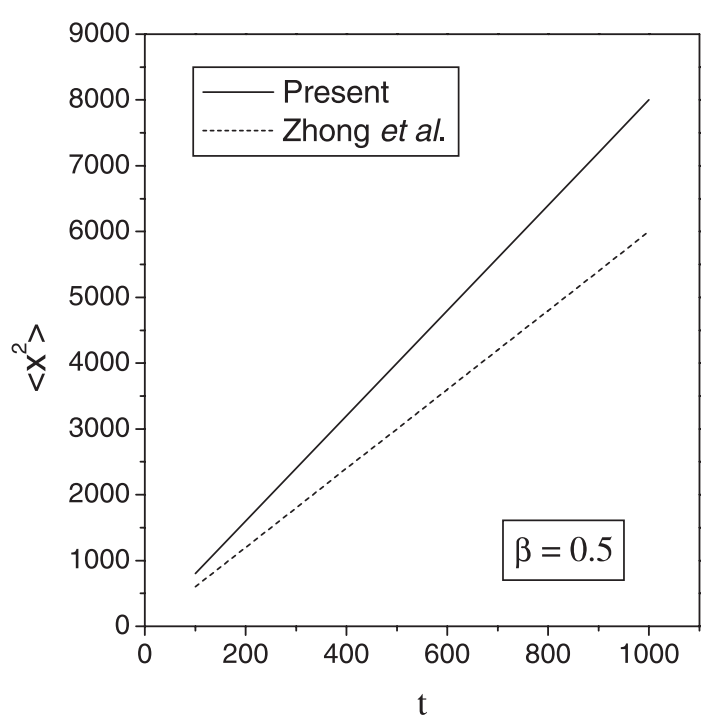

Fig. 4. The second moment as a function of time $t$ for $\beta=0.5$. The solid line gives our results while the dashed curve refers to the results of Zhong et al. [12].

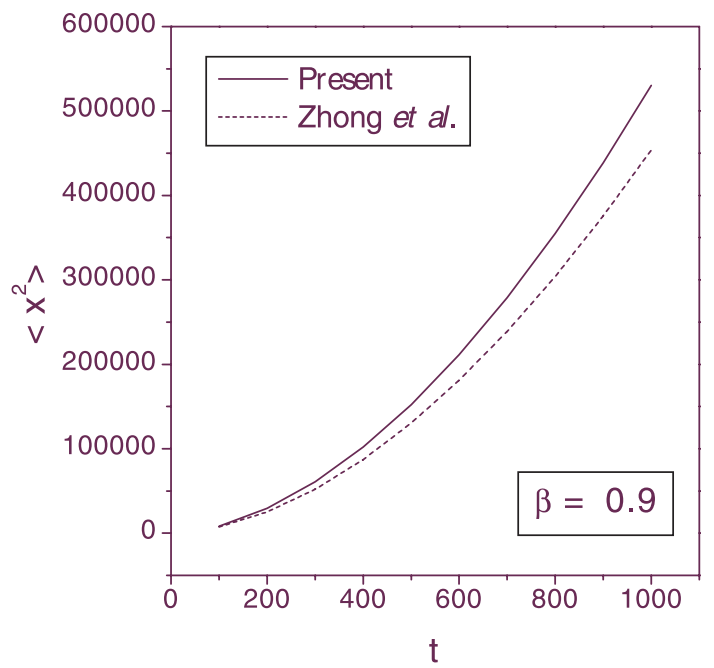

Fig. 5. The second moment as a function of time $t$ for $\beta=0.9$. The solid line gives our results while the dashed curve refers to the results of Zhong et al. [12].

The above integration is straight-forward and we get

$$
\left\langle x^{k}\right\rangle=\Gamma\left(1+\frac{k}{\gamma}\right)\left[\frac{t^{\beta}}{\left(f_{0} \beta^{\beta}(1-\beta)^{(1-\beta)}\right)}\right]^{k} .
$$

One can see that for $k=2$, equation (23) reduces to (20). It is interesting to note that though the probability in the present regime is a stretched exponential in contrast to the power-law behaviour that occurs in the case of $x<t^{\beta}$, the growth of moments is however given by a similar power law.

One may also look at the integrated wave packet which can be defined as

$$
G(x, t)=\int_{-x}^{x} d x^{\prime} P\left(x^{\prime}, t\right)
$$

and we get

$$
G(x, t)=\frac{1}{\omega^{\beta \gamma}}\left[1-e^{-\left(\frac{x}{\omega}\right)^{\gamma}}\right],
$$

while the simple solution of [12] gives

$$
G(x, t)=2 A w(1-\beta) \mathbf{P}\left((1+\beta),\left(\frac{x}{\omega}\right)^{\gamma}\right),
$$

where $\mathbf{P}(a, z)$ is the incomplete Gamma function defined as:

$$
\mathbf{P}(a, z)=\int_{0}^{z} d t e^{-t} t^{a-1} .
$$

In the limit, $x / w>>1$, Zhong et al.'s result becomes

$$
G(x, t)=\frac{\Gamma(1+\beta)}{\Gamma(1-\beta)}\left[1-\frac{1}{\Gamma(1+\beta)}\left(\frac{x}{w}\right)^{\beta \gamma} e^{-\left(\frac{|x|}{w}\right)^{\gamma}}\right],
$$

which in the limit $|x| \rightarrow \infty$ saturates to a constant in contrast to the power law behaviour obtained at short distances by Ketzmerick et al. Interestingly our result (25) at large $x$ yields a power law behaviour given by

$$
G(x, t) \sim t^{-\beta^{2} \gamma}
$$

which qualitatively describes a similar behaviour as obtained at short distances by Ketzmerick et al.

Again the spectral function

$$
S(x, s)=\frac{2}{\pi} \int d t \frac{\sin s t}{t} G(x, t)
$$

calculated with the solution of [12] also saturates to a constant at large $t$. However the more improved solution of the present work suggests a power law bahaviour

$$
S(x, s) \sim s^{\beta^{2} \gamma}
$$

again in conformity with the short distance behaviour suggested by Ketzmerick et al. [11].

Before we end, it would not be impertinent to worry about the exact differential equation satisfied by $P(x, t)$. From equation (11) one can immediately write an equation

$$
\frac{\partial^{2 \beta} P(x, t)}{\partial t^{2 \beta}}=\frac{1}{f_{0}^{2}} \frac{\partial^{2} P(x, t)}{\partial x^{2}}
$$

which holds of course for three values of $\beta$ : $\beta=0.5$ (classical limit), $\beta=0$ (localization limit), and $\beta=1$ (ballistic limit) and thus one can get exact solutions in these three cases and study all the relevant aspects.

In conclusion, we have given a fresh and a more rigorous look at the shape of the quantum diffusion front and obtained a more accurate solution of the probability distribution than that obtained by Zhong et al. [12]. We have also pointed out that under usual conditions the stretched exponential solution of Zhong et al. may be considered as fairly good but the range of the position coordinates over which it should be valid will be slightly different from the 
one given by them. We have shown that though the probability distribution might behave differently at small and large length scales, the moments of the distribution however follow similar power laws in both cases. The moment $\left\langle x^{2}\right\rangle$ is shown to increase quite strongly with increasing $\beta$. We have calculated the moment $\left\langle x^{2}\right\rangle$ using our probability density and also using the solution of Zhong et al. for the diffusive, ballistic and localization regimes and have shown that in all cases our solution leads to significant deviations as compared to the results obtained from Zhong et al.'s solution. We have also shown that the integrated wave packet exhibits a power law behaviour at long distance in conformity with the small distance result of Ketzmerick et al. [11] while the solution of Zhong et al. saturates to a constant. Finally we have shown that our solution also suggests a power law behaviour for the spectral function at long distance in conformity with the small distance behaviour predicted by Ketzmerick et al. [11]. Zhong et al.'s solution again predicts a saturation behaviour in this case. Thus, although our improved solution for the probability distribution apparently looks only marginally better that that of Zhong et al., it gives quantitatively different results in the case of moments and qualitatively different results in the case of integrated wave packet and the spectral function.
The author gratefully acknowledges the facilities provided by the Bilkent University, Ankara.

\section{References}

1. D. Shechtman et al., Phys. Rev. Lett. 53, 1951 (1984)

2. M. Schreiber, H. Grussbach, Phys. Rev. Lett. 67, 607 (1991)

3. B. Huckestein, L. Schweitzer, Phys. Rev. Lett. 72, 713 (1994)

4. R. Ketzmerick, G. Petschel, Geisel, Phys. Rev. Lett. 69, $695(1992)$

5. H. Hiramoto, S. Abe, J. Phys. Soc. Jpn 57, 230 (1988)

6. T. Geisel, R. Ketzmerick, G. Petschel, Phys. Rev. Lett. 66, $1651(1992)$

7. J.X. Zhong, R. Mosseri, J. Phys.: Condens. Matter 7, 8383 (1995)

8. B. Passaro, C. Sire, V.G. Benza, Phys. Rev. B 46, 13751 (1992)

9. I. Guarneri, Europhys. Lett. 10, 95 (1989); I. Guarneri, Europhys. Lett. 21, 729 (1993)

10. F. Piechon, Phys. Rev. Lett. 76, 4372 (1996)

11. R. Ketzmerick, K Kruse, S. Kraut, T. Geisel, Phys. Rev. Lett. 79, 1959 (1997)

12. J.X. Zhong et al., Phys. Rev. Lett. 86, 2485 (2001) 